\title{
Variable Structure Adaptive Fuzzy Control for A Class of Nonlinear Time Delay Systems
}

\author{
Changchun Hua*, Xinping Guan*, Guangren Duan** \\ *Institute of Electrical Engineering, Yanshan University, \\ Qinghuangdao, 066004, China \\ **Department of Control Engineering, \\ Harbin Institute of Technology, Heilongjiang Harbin, 150001, China
}

\begin{abstract}
In this paper, the problem of variable structure adaptive fuzzy control for a class of nonlinear time delay systems is investigated. Sliding surface dependent of the time delay is constructed based on the Lyapunov-Krasovskii method. Two adaptive fuzzy logic systems are proposed to approximate two unknown continuous nonlinear functions containing the current state and the delayed state respectively. The corresponding reaching law is designed, which can drive the state trajectory of the closed-system onto the sliding surface within limited time.
\end{abstract}

\section{INTRODUCTION}

Time-delay is often encountered in various engineering systems, such as electrical networks, turbojet engines, microwave oscillators, nuclear reactors, rolling mills, chemical processes, manual control, long transmission lines in pneumatic, and hydraulic systems, etc. Its existence is often a source of instability and poor performance. Therefore, the problem of stability analysis and robust control for dynamic time-delay systems has attracted considerable attention of a number of researchers over the past years [13, 14 and the references therein].

Fuzzy control has recently found extensive applications for a wide variety of industrial systems, and has attracted the attention of many control researchers due to its model free approach ([1] and [2]). Fuzzy control algorithms attempt to make use of information from human experts, which is generally represented by fuzzy terms, such as small, large, not very large or not very small, etc. More recently, an important adaptive fuzzy control system has been developed incorporated with the expert information systematically and the stability is guaranteed by theoretical analyses ([3] and [4]). An adaptive fuzzy system is a fuzzy logic system equipped with a training algorithm, in which the fuzzy logic system is constructed from a collection of fuzzy IF-THEN rules, and the training algorithm adjusts the parameters of the fuzzy logic system according to numerical input/output data.

Based on fuzzy logic systems which are capable of approximating, with arbitrary accuracy, any real continuous functions on a compact set, a globally stable adaptive controller is first synthesized from a collection of IF-THEN rules in [5]. In the existing literature on fuzzy control of nonlinear systems, fuzzy logic systems are used to approximate unknown nonlinear functions. Reference [6] investigated $H_{\infty}$ tracking problem of uncertain nonlinear SISO systems with external disturbance by using adaptive fuzzy controllers. Fuzzy indirect adaptive controller for a class of decentralized nonlinear systems is constructed in [7]. Paper [8] investigated robust adaptive fuzzy control for SISO nonlinear systems based on backstepping method. However, in the existing papers, few results on fuzzy control of time delay nonlinear systems are reported. Based on T-S fuzzy models, [15] was the first paper on the fuzzy control using Lyapunov-Krasovskii functional approach, while [16] used the Lyapunov-Razumikhin functional appraoch.

In this paper we consider the problem of robust variable structure adaptive fuzzy control for a class of nonlinear systems with time delay. As we know that a sliding mode control has attractive features such as fast response and good transient response. So we adopt the sliding mode control method in this paper. Fuzzy logic systems are also used as universal approximators of unknown nonlinear functions. The problem of designing both a linear sliding surface and reaching motion controller is investigated. A sliding surface is first designed by solving a Riccati inequality, which can guarantee that the sliding motion is exponentially stable dependent of the time delay. Then the reaching motion controller is designed, which can drive the state trajectory of the system onto the sliding surface within limited time.

The paper is organized as follows. The problem formulation is given in Section 2. A brief description of fuzzy logic systems is provided in Section 3. In Sections 4 and 5, the designs of the sliding surface and the reaching law are given respectively.

\section{PROBLEM FORMULATION}

Consider the following time delay system

$$
\begin{aligned}
\dot{x}= & (A+\Delta A(t)) x+\left(A_{d}+\Delta A_{d}(t)\right) x(t-d) \\
& +B(u+F(x, x(t-d), \sigma(t))) \\
& x(t)=\varphi(t), t \in\left[\begin{array}{ll}
-d & 0
\end{array}\right] .
\end{aligned}
$$

where $x \in R^{n}$ and $u \in R$ are the state and control input respectively, $d$ is the delay parameter, $A, B$ and $A_{d}$ are matrices with appropriate dimensions. $\Delta A(t)$ and $\Delta A_{d}(t)$ are time-varying uncertainties. $F(x, x(t-d), \sigma(t))$ is an 
unknown function containing uncertainties, where $\sigma(t)$ is a time varying disturbance parameter. In system (1), for simplicity we assume

$$
B=[0, \cdots, 0,1]^{T}
$$

and other matrices can be decomposed into the following

$$
\begin{aligned}
A & =\left(\begin{array}{ll}
A_{11} & A_{12} \\
A_{21} & A_{22}
\end{array}\right), \\
\Delta A(t) & =\left(\begin{array}{ll}
\Delta A_{11} & \Delta A_{12} \\
\Delta A_{21} & \Delta A_{22}
\end{array}\right), \\
A_{d} & =\left(\begin{array}{ll}
A_{d 11} & A_{d 12} \\
A_{d 21} & A_{d 22}
\end{array}\right), \\
\Delta A_{d} & =\left(\begin{array}{ll}
\Delta A_{d 11} & \Delta A_{d 12} \\
\Delta A_{d 21} & \Delta A_{d 22}
\end{array}\right)
\end{aligned}
$$

where $\quad A_{11}, \Delta A_{11}, A_{d 11}, \Delta A_{d 11} \in R^{(n-1) \times(n-1)}$, $A_{22}, \Delta A_{22}, A_{d 22}, \Delta A_{d 22} \in R$. Thus system (1) can be written as

$$
\begin{aligned}
\dot{x}_{1}= & \left(A_{11}+\Delta A_{11}\right) x_{1} \\
& +\left(A_{d 11}+\Delta A_{d 11}(t)\right) x_{1}(t-d) \\
& +\left(A_{12}+\Delta A_{12}\right) x_{2}+\left(A_{d 12}+\Delta A_{d 12}\right) x_{2}(t-d) \\
x_{1}(t)=\quad & \bar{\varphi}_{1}(t), \quad t \in\left[\begin{array}{ll}
-d & 0
\end{array}\right] \\
x_{2}= & \left(A_{21}+\Delta A_{21}\right) x_{1} \\
& +\left(A_{d 21}+\Delta A_{d 21}(t)\right) x_{1}(t-d) \\
& +\left(A_{22}+\Delta A_{22}\right) x_{2}+\left(A_{d 22}+\Delta A_{d 22}\right) x_{2}(t-d) \\
& +u+F(x, x(t-d), \sigma(t)) \\
x_{2}(t)= & \bar{\varphi}_{2}(t), \quad t \in\left[\begin{array}{ll}
-d & 0
\end{array}\right]
\end{aligned}
$$

where $x_{1} \in R^{n-1}, x_{2} \in R$. We introduce the following assumptions imposed on the system (1):

Assumption 1 The uncertainties $\Delta A_{11}(t), \Delta A_{12}(t)$, $\Delta A_{d 11}(t)$ and $\Delta A_{d 12}(t)$ satisfy

$$
\begin{aligned}
& {\left[\begin{array}{ll}
\Delta A_{11}(t) & \Delta A_{12}(t)
\end{array}\right]=D_{1} F_{1}(t)\left[\begin{array}{ll}
E_{11} & E_{12}
\end{array}\right]} \\
& {\left[\begin{array}{ll}
\Delta A_{d 11}(t) & \Delta A_{d 12}(t)
\end{array}\right]=D_{2} F_{2}(t)\left[\begin{array}{ll}
E_{21} & E_{22}
\end{array}\right]}
\end{aligned}
$$

where $\left\|F_{1}(t)\right\| \leq 1$, and $D_{1}, D_{2}, E_{11}, E_{12}, E_{21}$ and $E_{22}$ are some known matrices with proper dimensions. In addition

$$
\|\Delta A\| \leq \gamma_{1},\left\|\Delta A_{d}\right\| \leq \gamma_{2}
$$

Where positive scalars $\gamma_{1}$ and $\gamma_{2}$ are not required to be known.

Assumption $2 F(x, x(t-d), \sigma(t))$ satisfies the following decomposition

$$
\begin{aligned}
& F(x, x(t-d), \sigma(t)) \\
= & F_{1}(x)+F_{2}(x(t-d))+\Delta F(x(t), x(t-d), \sigma(t))
\end{aligned}
$$

where $F_{1}(x)$ and $F_{2}(x(t-d))$ are unknown continuous functions, $\Delta F(x(t), x(t-d), \sigma(t))$ is an uncertain function which is also unknown, but bounded

$|\Delta F(x(t), x(t-d), \sigma(t))| \leq \alpha_{0}+\alpha_{1}\|x(t)\|+\alpha_{2}\|x(t-d)\|$

where $\alpha_{0}, \alpha_{1}$ and $\alpha_{2}$ are unknown positive scalars.

Problem: Design a sliding surface to guarantee the sliding motion exponentially stable with a given decay rate $\gamma$ for system (1) and construct the reaching control law $u(t)$ to render the state trajectory of the closed-loop system convergent to the sliding surface in limited time.

\section{DESCRIPTION OF FUZZY LOGIC SYSTEMS}

A fuzzy system is a collection of fuzzy IF-THEN rules of the form:

$$
\begin{aligned}
R^{(j)}: & \text { IF } x_{1} \text { is } A_{1}^{j} \text { and } \cdots \text { and } x_{n} \text { is } A_{n}^{j} \\
& \text { THEN y is } B^{j} .
\end{aligned}
$$

By using the strategy of singleton fuzzification, product inference and center-average defuzzification, the output of the fuzzy system

$$
y(x)=\frac{\sum_{j=1}^{l} y^{j}\left(\prod_{i=1}^{n} \mu_{A_{i}^{j}}\left(x_{i}\right)\right)}{\sum_{j=1}^{l} \prod_{i=1}^{n} \mu_{A_{i}^{j}}\left(x_{i}\right)}
$$

where $\mu_{A_{i}^{j}}\left(x_{i}\right)$ is the membership function of linguistic variable $x_{i}$, and $y^{j}$ is the point in $R$ at which $\mu_{B^{j}}$ achieves its maximum value (assume that $\mu_{B^{i}}\left(y^{i}\right)=1$ ).

By introducing the concept of the fuzzy basic function vector $\zeta(x),(8)$ can be written as

$$
y(x)=\theta^{T} \zeta(x)
$$

where

$$
\theta=\left(\theta_{1}, \cdots, \theta_{l}\right)^{T}, \zeta(x)=\left(\zeta_{1}(x), \cdots, \zeta_{l}(x)\right)^{T}
$$

and $\zeta^{j}(x)$ is defined as

$$
\zeta_{j}(x)=\frac{\prod_{i=1}^{n} \mu_{A_{i}^{j}}\left(x_{i}\right)}{\sum_{j=1}^{l} \prod_{i=1}^{n} \mu_{A_{i}^{j}}\left(x_{i}\right)}
$$

In this paper we will take two universal fuzzy systems $\widehat{F}_{1}\left(x, \Theta_{1}\right)$ and $\widehat{F}_{2}\left(x(t-d), \Theta_{2}\right)$ with $x, x(t-d) \in U_{x}$ for some compact set $U_{x}$, to approximate the uncertain terms $\widetilde{F}_{1}(x(t))$ and $\widetilde{F}_{2}(x(t-d))$ defined in (34), where $\Theta_{1}$ and $\Theta_{2}$ contain tunable parameters. We construct the fuzzy rule bases of $\widehat{F}_{1}\left(x, \Theta_{1}\right)$ and $\widehat{F}_{2}\left(x(t-d), \Theta_{2}\right)$ consisting of the following rules: 


$$
\begin{gathered}
R_{F_{1}}^{j}: \text { IF } x_{1} \text { is } A_{1}^{j} \text { and } \cdots \text { and } x_{n} \text { is } A_{n}^{j} \\
\text { THEN } \widehat{F}_{1}\left(x, \Theta_{1}\right) \text { is } F_{1}^{j} . \\
R_{F_{2}}^{j} \quad: \quad \text { IF } x_{1}(t-d) \text { is } \bar{A}_{1}^{j} \text { and } \cdots \text { and } x_{n}(t-d) \text { is } \bar{A}_{n}^{j} \\
\text { THEN } \widehat{F}_{2}\left(x(t-d), \Theta_{2}\right) \text { is } F_{2}^{j} .
\end{gathered}
$$

and construct the following fuzzy systems

$$
\widehat{F}_{1}\left(x, \Theta_{1}\right)=\Theta_{1}^{T} \xi_{1}(x)
$$

and

$$
\widehat{F}_{2}\left(x(t-d), \Theta_{2}\right)=\Theta_{2}^{T} \xi_{2}(x(t-d))
$$

where $\xi_{1}(x)$ and $\xi_{2}(x(t-d))$ are fuzzy basis functions. According to the universal approximation theorem there exist optimal approximation parameters $\Theta_{1}^{*}$ and $\Theta_{2}^{*}$ [3], such that $\xi_{1}(x)^{T} \Theta_{1}^{*}$ and $\xi_{2}(x(t-d))^{T} \Theta_{2}^{*}$ can approximate $\widetilde{F}_{1}(x)$ and $\widetilde{F}_{2}(x(t-d))$ to any desired degree. The parameters $\Theta_{1}^{*}$ and $\Theta_{2}^{*}$ are defined as follows

$$
\begin{gathered}
\Theta_{1}^{*}=\arg \min _{\Theta_{1} \in \Omega_{F_{1}}}\left(\sup _{x \in \Omega_{x}}\left|\widehat{F}_{1}\left(x, \Theta_{1}\right)-\widetilde{F}_{1}(x)\right|\right) \\
\Theta_{2}^{*}=\arg \min _{\Theta_{2} \in \Omega_{F_{2}}}\left(\sup _{x(t-d) \in \Omega_{x}}\left|\begin{array}{c}
\widehat{F}_{2}\left(x(t-d), \Theta_{2}\right) \\
-\widetilde{F}_{2}(x(t-d))
\end{array}\right|\right)
\end{gathered}
$$

where $\Omega_{F_{1}}, \Omega_{F_{2}}$ and $\Omega_{x}$ denote the sets of suitable bounds on $\Theta_{1}, \Theta_{2}$ and $x$, respectively. We assume that $\Theta_{1}, \Theta_{2}$ and $x$ never reach the boundaries of $\Omega_{F_{1}}, \Omega_{F_{2}}$ and $\Omega_{x}$. And the minimum approximation error satisfies the following assumption

Assumption 3 The approximation error between $\widetilde{F}_{1}(x), \widetilde{F}_{2}(x(t-d))$ and $\xi_{1}(x)^{T} \Theta_{1}^{*}, \xi_{2}(x(t-d))^{T} \Theta_{2}^{*}$ satisfies the following inequality

$$
\begin{aligned}
& \left|\begin{array}{c}
\widetilde{F}_{1}(x)-\xi_{1}(x)^{T} \Theta_{1}^{*}+\widetilde{F}_{2}(x(t-d)) \\
-\xi_{2}(x(t-d))^{T} \Theta_{2}^{*}
\end{array}\right| \\
& \leq \quad \hbar_{0}+\hbar_{1}\|x\|+\hbar_{2}\|x(t-d)\|
\end{aligned}
$$

where $\hbar_{0}, \hbar_{1}$ and $\hbar_{2}$ are unknown scalars. These two parameters $\Theta_{1}^{*}$ and $\Theta_{2}^{*}$ will be learned by using the adaptive algorithms.

\section{SLIDING SURFACE DESIGN}

Without loss of generality, we suppose that the sliding surface is

$$
S=\widetilde{C} x=\left[\begin{array}{ll}
-C & 1
\end{array}\right]\left[\begin{array}{ll}
x_{1}^{T} & x_{2}
\end{array}\right]^{T}=\left(-C x_{1}+x_{2}\right)=0
$$

where $C \in R^{n-1}$. Hence, substituting $x_{2}=C x_{1}$ into (3) gives the sliding motion

$$
\begin{aligned}
\dot{x}_{1}= & \left(A_{11}+\Delta A_{11}\right) x_{1} \\
& +\left(A_{d 11}+\Delta A_{d 11}(t)\right) x_{1}(t-d) \\
& +\left(A_{12}+\Delta A_{12}\right) C x_{1} \\
& +\left(A_{d 12}+\Delta A_{d 12}\right) C x_{1}(t-d) \\
x_{1}(t)= & \bar{\varphi}_{1}(t), \quad t \in\left[\begin{array}{ll}
-d & 0
\end{array}\right]
\end{aligned}
$$

If system (13) is exponentially stable dependent of the delay, the sliding motion (13) will be exponentially stable dependent of the delay.

Theorem 1 Consider system (13), if assumption 1 is met and the following inequality is satisfied

$$
\begin{aligned}
& P\left(A_{11}+\gamma I\right)+\left(A_{11}+\gamma I\right)^{T} P \\
& +\varepsilon^{-1} P D_{1} D_{1}^{T} P+\varepsilon E_{11}^{T} E_{11} \\
& +\varepsilon^{-1} e^{2 \gamma d} P A_{d 12} A_{d 12}^{T} P+e^{2 \gamma d} P A_{d 11} X A_{d 11}^{T} P+X^{-1} \\
& +\varepsilon^{-1} e^{2 \gamma d} P D_{2} D_{2}^{T} P+\varepsilon E_{21}^{T} E_{21} \\
& -\left[\varepsilon\left(1+E_{12}^{T} E_{12}+E_{22}^{T} E_{22}\right)\right]^{-1} \\
& \left(P A_{12}+\varepsilon E_{11}^{T} E_{12}+\varepsilon E_{21}^{T} E_{22}\right) \\
& \left(P A_{12}+\varepsilon E_{11}^{T} E_{12}+\varepsilon E_{21}^{T} E_{22}\right)^{T} \\
< & 0
\end{aligned}
$$

where $P$ and $X$ are positive matrices, $\varepsilon$ is a positive scalar, then the following sliding surface

$$
\begin{aligned}
S= & {\left[\varepsilon\left(1+E_{12}^{T} E_{12}+E_{22}^{T} E_{22}\right)\right]^{-1} } \\
& \left(P A_{12}+\varepsilon E_{11}^{T} E_{12}+\varepsilon E_{21}^{T} E_{22}\right)^{T} x_{1} \\
& +x_{2}
\end{aligned}
$$

will render the sliding motion exponentially stable dependent of the delay with a given decay rate $\gamma$.

Proof: For system (13), we choose the following coordinate transformation

$$
z(t)=e^{\gamma t} x_{1}(t)
$$

we can obtain

$$
\begin{aligned}
\dot{z}= & \gamma e^{\gamma t} x_{1}+e^{\gamma t} \dot{x} \\
= & \left(A_{11}+A_{12} C+\gamma I+\Delta A_{11}+\Delta A_{12} C\right) z \\
& +e^{\gamma d}\left(A_{d 11}+A_{d 12} C+\Delta A_{d 11}+\Delta A_{d 12} C\right) z(t-d)
\end{aligned}
$$

As we know, if system (16) is robust asymptotically stable, system (13) will be exponential stable with a given attenuance $\gamma$ dependent of the delay.

For system (16), let us define the following LyapunovKrasovskii function

$$
\begin{aligned}
V(z)= & z^{T} P z+\int_{t-d}^{t} \varepsilon z^{T}(v) \\
& {\left[\begin{array}{c}
\left(E_{21}+E_{22} C\right)^{T}\left(E_{21}+E_{22} C\right) \\
+X^{-1}+C^{T} C
\end{array}\right] z(v) d v }
\end{aligned}
$$


Then the time derivative along the state trajectory of the system (16) is

$$
\begin{aligned}
\dot{V}= & 2 z^{T} P\left(\begin{array}{c}
A_{11}+A_{12} C+\gamma I \\
+\Delta A_{11}+\Delta A_{12} C
\end{array}\right) z \\
& +2 e^{\gamma d} z^{T} P\left(\begin{array}{c}
A_{d 11}+A_{d 12} C \\
+\Delta A_{d 11}+\Delta A_{d 12} C
\end{array}\right) z(t-d) \\
& +\varepsilon z^{T}(t)\left[\begin{array}{c}
\left(E_{21}+E_{22} C\right)^{T}\left(E_{21}+E_{22} C\right) \\
+X^{-1}+C^{T} C
\end{array}\right] z(t) \\
& -\varepsilon z^{T}(t-d)\left[\begin{array}{c}
\left(E_{21}+E_{22} C\right)^{T}\left(E_{21}+E_{22} C\right) \\
+X^{-1}+C^{T} C
\end{array}\right] \\
& z(t-d)
\end{aligned}
$$

Note

$$
\begin{aligned}
& 2 z^{T} P\left(\Delta A_{11}+\Delta A_{12} C\right) z \\
& =2 z^{T} P D_{1} F_{1}(t)\left(E_{11}+E_{12} C\right) z \\
& \leq \varepsilon_{1}^{-1} z^{T} P D_{1} D_{1}^{T} P z \\
& +\varepsilon_{1} z^{T}\left(E_{11}+E_{12} C\right)^{T}\left(E_{11}+E_{12} C\right) z \\
& 2 e^{\gamma d} z^{T} P\left(A_{d 12} C+\Delta A_{d 11}+\Delta A_{d 12} C\right) z(t-d) \\
& \leq \varepsilon_{2}^{-1} e^{2 \gamma d} z^{T} P A_{d 12} A_{d 12}^{T} P z \\
& +\varepsilon_{2} z(t-d)^{T} C^{T} C z(t-d)+\varepsilon_{3}^{-1} e^{2 \gamma d} z^{T} P D_{2} D_{2}^{T} P z \\
& +\varepsilon_{3} z(t-d)^{T}\left(E_{21}+E_{22} C\right)^{T}\left(E_{21}+E_{22} C\right) z(t-d) \\
& 2 e^{\gamma d} z^{T} P A_{d 11} z(t-d) \\
& \leq e^{2 \gamma d} z^{T} P A_{d 11} X A_{d 11}^{T} P z+z(t-d)^{T} X^{-1} z(t-d)
\end{aligned}
$$

where $X$ is a positive matrix, $\varepsilon_{1}, \varepsilon_{2}$ and $\varepsilon_{3}$ are positive scalars. For simplicity we choose $\varepsilon_{1}=\varepsilon_{2}=\varepsilon_{3}=\varepsilon$, then by substituting (18),(19) and (20) into (17), we get

$$
\begin{aligned}
\dot{V} \leq & 2 z^{T} P\left(A_{11}+A_{12} C+\gamma I\right) z+\varepsilon^{-1} z^{T} P D_{1} D_{1}^{T} P z \\
& +\varepsilon z^{T}\left(E_{11}+E_{12} C\right)^{T}\left(E_{11}+E_{12} C\right) z \\
& +\varepsilon^{-1} e^{2 \gamma d} z^{T} P A_{d 12} A_{d 12}^{T} P z+\varepsilon z^{T} C^{T} C z \\
& +e^{2 \gamma d} z^{T} P A_{d 11} X A_{d 11}^{T} P z+z^{T} X^{-1} z \\
& +\varepsilon^{-1} e^{2 \gamma d} z^{T} P D_{2} D_{2}^{T} z \\
& +\varepsilon z^{T}\left(E_{21}+E_{22} C\right)^{T}\left(E_{21}+E_{22} C\right) z \\
= & 2 z^{T} P\left(A_{11}+\gamma I\right) z+\varepsilon^{-1} z^{T} P D_{1} D_{1}^{T} P z \\
& +\varepsilon z^{T} E_{11}^{T} E_{11} z+\varepsilon^{-1} e^{2 \gamma d} z^{T} P A_{d 12} A_{d 12}^{T} P z \\
& +e^{2 \gamma d} z^{T} P A_{d 11} X A_{d 11}^{T} P z+z^{T} X^{-1} z \\
& +\varepsilon^{-1} e^{2 \gamma d} z^{T} P D_{2} D_{2}^{T} z+\varepsilon z^{T} E_{21}^{T} E_{21} z \\
& +\varepsilon z^{T} C^{T}\left(1+E_{12}^{T} E_{12}+E_{22}^{T} E_{22}\right) C z \\
& +2 z^{T}\left(P A_{12}+\varepsilon E_{11}^{T} E_{12}+\varepsilon E_{21}^{T} E_{22}\right) C z
\end{aligned}
$$

\section{in limited time.}

For system (1), consider the following controller

$$
u=u_{1}+u_{2}+u_{3}
$$

where

$$
\begin{gathered}
u_{1}=-\Theta_{1}^{T} \xi_{1}(x)-\Theta_{2}^{T} \xi_{2}(x(t-d)) \\
u_{2}=-\vartheta_{0} \operatorname{sign}(\widetilde{C} x)-\vartheta_{1}\|x\| \operatorname{sign}(\widetilde{C} x) \\
-\vartheta_{2}\|x(t-d)\| \operatorname{sign}(\widetilde{C} x) \\
u_{3}=-\beta \widetilde{C} x
\end{gathered}
$$

where $\beta$ is a positive scalar, $\Theta_{1}, \Theta_{2}, \vartheta_{0}, \vartheta_{1}$ and $\vartheta_{2}$ are adaptive parameters, whose adaptive laws are as follows

$$
\begin{gathered}
\dot{\Theta}_{1}=\Gamma_{1} \widetilde{C} x \xi_{1}(x) \\
\dot{\Theta}_{2}=\Gamma_{2} \widetilde{C} x \xi_{2}(x(t-d)) \\
\dot{\vartheta}_{0}=k_{0}|\widetilde{C} x|
\end{gathered}
$$

So if we let 


$$
\begin{gathered}
\dot{\vartheta}_{1}=k_{1}|\widetilde{C} x|\|x\| \\
\dot{\vartheta}_{2}=k_{2}|\widetilde{C} x|\|x(t-d)\|
\end{gathered}
$$

where $\Gamma_{1}$ and $\Gamma_{2}$ are positive matrices with proper dimensions, $k_{1}, k_{2}$ and $k_{3}$ are positive scalars.

In the above controller, $u_{1}$ contains two fuzzy logic systems used to approximate the unknown nonlinear functions, $u_{2}$ is an adaptive controller used to compensate for the timevarying uncertainties and the approximation error, and $u_{3}$ is used to further guarantee that the sliding function $S(\cdot)$ asymptotically converges to zero.

Theorem 2 Given system (1) with the sliding surface (15), the above reaching law (23) will drive the trajectory of the closed-loop system onto the sliding surface in limited time.

Proof: For system (1), define the following Lyapunov function

$$
\begin{aligned}
V(x)= & \frac{1}{2}\left[S^{2}+\bar{\Theta}_{1}^{T} \Gamma_{1}^{-1} \bar{\Theta}_{1}+\bar{\Theta}_{2}^{T} \Gamma_{2}^{-1} \bar{\Theta}_{2}\right. \\
& \left.+k_{0}^{-1} \bar{\vartheta}_{0}^{2}+k_{1}^{-1} \bar{\vartheta}_{1}^{2}+k_{2}^{-1} \bar{\vartheta}_{2}^{2}\right]
\end{aligned}
$$

where $\bar{\Theta}_{1}=\Theta_{1}^{*}-\Theta_{1}, \bar{\Theta}_{2}=\Theta_{2}^{*}-\Theta_{2}, \bar{\vartheta}_{0}=\vartheta_{0}^{*}-\vartheta_{0}, \bar{\vartheta}_{1}=$ $\vartheta_{1}^{*}-\vartheta_{1}$ and $\bar{\vartheta}_{2}=\vartheta_{2}^{*}-\vartheta_{2}$.

The time derivative along the state trajectory of the system (1) is

$$
\begin{aligned}
\dot{V}= & \dot{S S}+\bar{\Theta}_{1}^{T} \Gamma_{1}^{-1} \bar{\Theta}_{1}+\bar{\Theta}_{2}^{T} \Gamma_{2}^{-1} \bar{\Theta}_{2} \\
& +k_{0}^{-1} \bar{\vartheta}_{0} \bar{\vartheta}_{0}+k_{1}^{-1} \bar{\vartheta}_{1} \bar{\vartheta}_{1}+k_{2}^{-1} \bar{\vartheta}_{2} \bar{\vartheta}_{2}
\end{aligned}
$$

As we know

$$
\begin{aligned}
\dot{S S=} & \widetilde{C} x \widetilde{C} x \\
= & \widetilde{C} x \widetilde{C}\left[(A+\Delta A(t)) x+\left(A_{d}+\Delta A_{d}(t)\right) x(t-d)\right. \\
& +B(u+F(x, x(t-d), \sigma(t)))] \\
= & \widetilde{C} x\left[\widetilde{C}\left((A+\Delta A(t)) x+\left(A_{d}+\Delta A_{d}(t)\right) x(t-d)\right)\right. \\
& +u+F(x, x(t-d), \sigma(t))]
\end{aligned}
$$

Substituting the controller (22) into the above equality, we can obtain

$$
\begin{aligned}
& \dot{S S} \\
= & \widetilde{C} x\left[\widetilde{C}\left((A+\Delta A(t)) x+\left(A_{d}+\Delta A_{d}(t)\right) x(t-d)\right)\right. \\
& \left.+u_{1}+u_{2}+u_{3}+F(x, x(t-d), \sigma(t))\right]
\end{aligned}
$$

The unknown nonlinear continuous functions are defined as follows

$$
\begin{aligned}
\widetilde{F}_{1}(x) & =\widetilde{C} A x+F_{1}(x) \\
\widetilde{F}_{2}(x(t-d)) & =\widetilde{C} A_{d} x(t-d)+F_{2}(x(t-d))
\end{aligned}
$$

From the knowledge of section 3, we use two fuzzy logic systems $\widehat{F}_{1}\left(x, \Theta_{1}\right)=\Theta_{1}^{T} \xi_{1}(x)$ and $\widehat{F}_{2}\left(x(t-d), \Theta_{2}\right)=$ $\Theta_{2}^{T} \xi_{2}(x(t-d))$, to approximate $\widetilde{F}_{1}(x)$ and $\widetilde{F}_{2}(x(t-d))$, substituting (23) into (33) we further get

$$
\begin{aligned}
\dot{S S=} & \widetilde{C} x\left[\widetilde{C} \Delta A(t) x+\widetilde{C} \Delta A_{d}(t) x(t-d)\right. \\
& +\widetilde{F}_{1}(x)-\Theta_{1}^{* T} \xi_{1}(x) \\
& +\widetilde{F}_{2}(x(t-d))-\Theta_{2}^{* T} \xi_{2}(x(t-d)) \\
& +\left(\Theta_{1}^{*}-\Theta_{1}\right)^{T} \xi_{1}(x) \\
& +\left(\Theta_{2}^{*}-\Theta_{2}\right)^{T} \xi_{2}(x(t-d)) \\
& \left.+u_{2}+u_{3}+\Delta F(x, x(t-d), \sigma(t))\right]
\end{aligned}
$$

From assumptions 2 and 3 , it is easy to obtain that there exist unknown positive scalars $\vartheta_{0}^{*}, \vartheta_{1}^{*}$ and $\vartheta_{2}^{*}$ satisfying the following inequality

$$
\begin{aligned}
& \widetilde{C} x(\Delta F(x, x(t-d), \sigma(t)) \\
& +\widetilde{C} \Delta A_{d}(t) x(t-d)+\widetilde{F}_{1}(x) \\
& -\Theta_{1}^{* T} \xi_{1}(x)+\widetilde{C} \Delta A(t) x \\
& \left.+\widetilde{F}_{2}(x(t-d))-\Theta_{2}^{* T} \xi_{2}(x(t-d))\right) \\
\leq & |\widetilde{C} x|\left[\vartheta_{0}^{*}+\vartheta_{1}^{*}\|x\|+\vartheta_{2}^{*}\|x(t-d)\|\right]
\end{aligned}
$$

Substitute (24), (25) and (36) into (35), we obtain

$$
\begin{aligned}
\dot{S S} \leq & |\widetilde{C} x|\left[\begin{array}{c}
\left(\vartheta_{0}^{*}-\vartheta_{0}\right)+\left(\vartheta_{1}^{*}-\vartheta_{1}\right)\|x\| \\
+\left(\vartheta_{2}^{*}-\vartheta_{2}\right)\|x(t-d)\|
\end{array}\right] \\
& +\left[\begin{array}{c}
\left(\Theta_{1}^{*}-\Theta_{1}\right)^{T} \xi_{1}(x) \\
+\left(\Theta_{2}^{*}-\Theta_{2}\right)^{T} \xi_{2}(x(t-d))
\end{array}\right] \widetilde{C} x \\
& -\beta S^{2}
\end{aligned}
$$

So we can get the following inequality by substituting (37) and (26-30) into (32)

$$
\begin{aligned}
\dot{V}= & S \dot{S}+\bar{\Theta}_{1}^{T} \Gamma_{1}^{-1} \bar{\Theta}_{1}+\bar{\Theta}_{2}^{T} \Gamma_{2}^{-1} \bar{\Theta}_{2} \\
& +k_{0}^{-1} \bar{\vartheta}_{1} \bar{\vartheta}_{1}+k_{1}^{-1} \bar{\vartheta}_{2} \bar{\vartheta}_{2}+k_{2}^{-1} \bar{\vartheta}_{3} \bar{\vartheta}_{3} \\
\leq & -\beta S^{2}
\end{aligned}
$$

From (38), it is easy to see that the reaching law (22) guarantees that the trajectory of system (1) is driven onto the sliding surface in limited time.

In the control process, we assume that $\Theta_{1}, \Theta_{2}$ never reach the boundary of $\Omega_{F_{1}}, \Omega_{F_{2}}$ defined as follows

$$
\begin{aligned}
& \Omega_{F_{1}}=\left\{\Theta_{1} \mid\left\|\Theta_{1}\right\| \leq M_{1}\right\} \\
& \Omega_{F_{2}}=\left\{\Theta_{2} \mid\left\|\Theta_{2}\right\| \leq M_{2}\right\}
\end{aligned}
$$

In order to guarantee the boundedness of the adaptive parameters $\Theta_{1}, \Theta_{2}$, we introduce the projection operator [5] to restrict them in the closed sets $\Omega_{F_{1}}, \Omega_{F_{2}}$. The parameter adaptive law (26) and (27) can be changed into 


$$
\dot{\Theta}_{1}=\left\{\begin{array}{c}
\Gamma_{1} \widetilde{C} x \xi_{1}(x) \text { If }\left\|\Theta_{1}\right\|<M_{1} \\
\text { or }\left(\left\|\Theta_{1}\right\|=M_{1} \text { and } \widetilde{C} x \Theta_{1}^{T} \xi_{1}(x) \leq 0\right) \\
P_{r}\left[\Gamma_{1} \widetilde{C} x \xi_{1}(x)\right] \text { If }\left\|\Theta_{1}\right\|=M_{1} \\
\text { and } \left.\widetilde{C} x \Theta_{1}^{T} \xi_{1}(x)>0\right)
\end{array}\right.
$$

and

$$
\dot{\Theta}_{2}=\left\{\begin{array}{c}
\Gamma_{2} \widetilde{C} x \xi_{2}(x(t-d)) \text { If }\left\|\Theta_{2}\right\|<M_{2} \\
\text { or }\left(\left\|\Theta_{2}\right\|=M_{2} \text { and } \widetilde{C} x \Theta_{2}^{T} \xi_{2}(x(t-d)) \leq 0\right) \\
\operatorname{Pr}_{r}\left[\Gamma_{2} \widetilde{C} x \xi_{2}(x(t-d))\right] \text { If }\left\|\Theta_{2}\right\|=M_{2} \\
\text { and } \left.\widetilde{C} x \Theta_{2}^{T} \xi_{2}(x(t-d))>0\right)
\end{array}\right.
$$

where $P_{r}$ is determined by the following equations

$$
\begin{aligned}
P_{r}\left[\Gamma_{1} \widetilde{C} x \xi_{1}(x)\right]=\Gamma_{1} \widetilde{C} x \xi_{1}(x)-\Gamma_{1} \frac{\widetilde{C} x \xi_{1}^{T}(x) \Theta_{1}}{\left\|\Theta_{1}\right\|^{2}} \Theta_{1} \\
\quad P_{r}\left[\Gamma_{2} \widetilde{C} x \xi_{2}(x(t-d))\right] \\
=\Gamma_{2} \widetilde{C} x \xi_{2}(x(t-d))-\Gamma_{2} \frac{\widetilde{C} x \xi_{2}^{T}(x(t-d)) \Theta_{2}}{\left\|\Theta_{2}\right\|^{2}} \Theta_{2}
\end{aligned}
$$

We can find that the theorem can also be easily proved by utilizing the adaptive laws (40) and (41) instead of (26) and (27). Here the proof is omitted.

\section{CONCLUSION}

This paper investigates the problem of variable structure adaptive fuzzy control for a class of nonlinear systems with time delays. The sliding surface and the corresponding reaching law are designed. Different from the existing literature, the sliding surface in this paper is dependent on the time delay, and the sliding motion is exponentially stable. During the design of reaching law, fuzzy logic systems are adopted to approximate unknown functions. The constructed reaching law can drive the state trajectory of the system onto the sliding surface in limited time.

\section{Acknowledgements}

This work is supported in part by NSF of China (No. 60174010 and No. 60274023).

\section{REFERENCES}

[1] L. A. Zadeh, Fuzzy Sets, Information Contr., vol. 8, pp.338-353, 1965.

[2] M. Jamshidi, N. Vadiee, and T. J. Ress, Fuzzy Logic and Control. Englewood Cliffs, NJ: Prentice-Hall, 1993.

[3] L. X. Wang, Adaptive Fuzzy Systems and Control: Design and stability Analysis. Englewood Cliffs, NJ: Prentice-Hall, 1994.

[4] L. X. Wang, and J. M. Mendel, Fuzzy bass function, universal approximation, and orthogonal least square learning, IEEE Trans. Neural Networks, vol. 3, no. 5, pp.807-814, 1992.

[5] L. X. Wang, Stable adaptive fuzzy control of nonlinear systems, IEEE Trans. Fuzzy Systems, Vol. 1, no. 2, 146-155, 1993.

[6] B. Chen, C. Lee and Y. Chang, $H^{\infty}$ tracking design of uncertain nonlinear siso systems: adaptive fuzzy approach. IEEE Trans. Fuzzy Systems vol 4, 32-43, 1996.
[7] S. Tong and T. Chai, Fuzzy indirect adaptive control for a class of decentralized nonlinear systems. International Journal of Systems Science, vol. 29, no. 2: 149-157, 1998.

[8] H. Lee and M. Tomizuka, Robust adaptive control using a universal approximator for SISO nonlinear systems. IEEE Trans. Fuzzy Systems, vol 8, no. 1, 95-106, 2000.

[9] J. Wang, A. B. Rad and P. T. Chan, Indirect adaptive fuzzy sliding mode control: Part I: fuzzy switching. Fuzzy sets and Systems, no.122, 21-30, 2001.

[10] P. T. Chan, A. B. Rad and J. Wang, Indirect adaptive fuzzy sliding mode control: Part II: parameter projection and supervisory control. Fuzzy sets and Systems, no.122, 31-43, 2001.

[11] J. Hu, J. Chu and H. Su, SMVSC for a class of time-delay uncertain systems with mismatching uncertainties. IEE Proc. -Control Theory Appl, vol.147, No. 6: 687-693, 2000.

[12] E. K. Reyad, Variable structure robust control uncertain time-delay systems. Automatica, vol. 34, No. 3: pp.327-332, 1998.

[13] S.I. Niculescu, E. I. Verriest, L. Dugard, and J. M. Dion, Stability and robust stability of time-delay systems: A guided tour in stability and control of time-delay systems, L. Duard and E. I. Verriest, Eds. London, U.K.: SpringerVerlag, pp.1-71, 1997.

[14] K. Gu and S.I. Niculescu, Further remarks on additional dynamics in various model transformations of linear delay systems. IEEE Trans. Autom. Contr., No. 3, vol. 46, pp. 497-500, 2001.

[15] Y.-Y. Cao and P.M. Frank, Stability analysis and synthesis of nonlinear time-delay systems via linear Takagi-Sugeno fuzzy models, Fuzzy sets and Systems, vol.124(12), 213-229,2001.

[16] Y.-Y. Cao and P.M. Frank, Analysis and synthesis of nonlinear time-delay systems via fuzzy control approach. IEEE Trans. Fuzzy systems, vol.8(2), 200-211, 2000. 